\title{
Semi-Active Control of Vehicle Seat Suspension System with Magnetorheological Damper
}

\author{
Haijun Xing ${ }^{1, a}$, Shaopu Yang ${ }^{1, b}$ and Yongjun Shen ${ }^{1, c}$ \\ ${ }^{1}$ School of Mechanical Engineering, Shijiazhuang Tiedao University, Shijiazhuang, Hebei 050043, \\ P.R. China \\ axinghj@stdu.edu.cn, byangsp@stdu.edu.cn, 'shenyongjun@126.com
}

Keywords: Semi-active Control, Seat Suspension, Magnetorheological Damper, LQR Control

Abstract. This research aims at the vibration control of vehicle seat suspension system. A three degree of freedom quarter vehicle model is used for semi-active control system in which a magnetorheological damper (MRD) is installed at the position between the vehicle body and the seat. A fully active linear quadratic regulator (LQR) control strategy is used to determine the optimized control force which is then matched by MRD to compute the semi-active control result. Computation result proves that semi-active control with MRD can alleviate the vehicle seat acceleration to improve ride comfort.

\section{Introduction}

There are three types of vehicle suspension systems, namely passive control suspension system, active control suspension system, and semi-active control suspension system[1]. In passive suspension system, the stiffness and damping parameters are fixed and are effective over a certain range of frequencies. In active suspension system, an actuator is used to supply adapting force to adapt to changing road conditions. High cost, complexity, need for an external energy source and difficulty in control hardware implementation restrict the development of active suspension system. The semi-active control suspension system is an alternative to the active suspension system and can combine the advantages of both passive and active suspension systems. In semi-active control suspension system, MRD whose damping force can be easy adjusted by changing magnetic fields have been considered by a number of researchers [2-5].

In this paper, A three degree of freedom quarter vehicle model in which the damping force between the seat and vehicle body is supplied by a MRD is used to calculate the control effect of a semi-active seat suspension system. In this semi-active seat suspension system, the optimized control force is determined by a fully active linear quadratic regulator (LQR) control strategy and the actual damping force the MRD can realized.

\section{Active Control Model of LQR Control Strategy}

Three Degree of Freedom Quarter Vehicle model. The three degree of freedom quarter vehicle model is shown in Fig. 1 and the equations of motion of the model are given by

$$
\begin{aligned}
& m_{1} \ddot{z}_{1}=k_{1}\left(z_{0}-z_{1}\right)-k_{2}\left(z_{1}-z_{2}\right)-c_{2}\left(\dot{z}_{1}-\dot{z}_{2}\right) \\
& m_{2} \ddot{z}_{2}=k_{2}\left(z_{1}-z_{2}\right)-k_{3}\left(z_{2}-z_{3}\right)+c_{2}\left(\dot{z}_{1}-\dot{z}_{2}\right)-F_{D 3} \\
& m_{3} \ddot{z}_{3}=k_{3}\left(z_{2}-z_{3}\right)+F_{D 3}
\end{aligned}
$$

In Fig. 1 and Esq. (1)-(3), $m_{1}, m_{2}$ and $m_{3}$ are masses of tyre, vehicle body and seat (including rider's mass) respectively, $k_{1}, k_{2}$ and $k_{3}$ are stiffnesses of tyre, vehicle body suspension and seat suspension respectively, $C_{2}$ is the damping coefficient of linear viscous damper connecting tyre and vehicle body, $z_{1}, z_{2}$ and $z_{3}$ are displacements of tyre, vehicle body and seat respectively, $z_{0}$ is the external road excitation. In active control system, $F_{D 3}$ is the optimized control force determined by the control strategy and can realized by an actuator, in semi-active control system, $F_{D 3}$ is realized by a MRD, and in passive control system, $F_{D 3}=C_{3}\left(\dot{z}_{2}-\dot{z}_{3}\right)$ where $C_{3}$ is the damping coefficient of linear viscous damper connecting seat and vehicle body. 


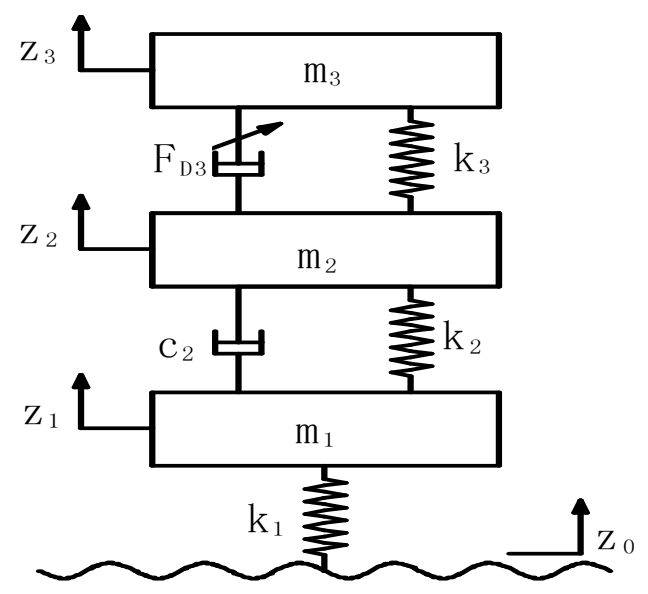

Fig. 1. Three degree of freedom quarter vehicle model

LQR Control Strategy. Defining the state vector $X=\left[\begin{array}{llllll}x_{1} & x_{2} & x_{3} & x_{4} & x_{5} & x_{6}\end{array}\right]^{T}=\left[\begin{array}{lll}z_{0}-z_{1} & \dot{z}_{1}\end{array}\right.$ $\left.z_{1}-z_{2} \quad \dot{z}_{2} \quad z_{2}-z_{3} \dot{z}_{3}\right]^{T}$ and the output vector $Y=\left[\begin{array}{llllll}y_{1} & y_{2} & y_{3} & y_{4} & y_{5} & y_{6}\end{array}\right]=\left[\begin{array}{llll}x_{1} & \dot{x}_{2} & x_{3}\end{array}\right.$ $\left.\begin{array}{lll}\dot{x}_{4} & x_{5} & \dot{x}_{6}\end{array}\right]$, Eqs. (1)- (3) and the output vector $Y$ can be written in matrix form as

$$
\begin{aligned}
& \dot{X}=A X+B U+G W \\
& Y=C X+D U
\end{aligned}
$$

where $A, B, U G$ and $W$ are system matrix, control distribution vector, control vector, excitation distribution vector and excitation vector. $A, B, U G, W, C$ and $D$ are given as follows:

$$
\begin{aligned}
& A=\left[\begin{array}{cccccc}
0 & -1 & 0 & 0 & 0 & 0 \\
\frac{k_{1}}{m_{1}} & \frac{-c_{2}}{m_{1}} & \frac{-k_{2}}{m_{1}} & \frac{c_{2}}{m_{1}} & 0 & 0 \\
0 & 1 & 0 & -1 & 0 & 0 \\
0 & \frac{c_{2}}{m_{2}} & \frac{k_{2}}{m_{2}} & \frac{-c_{2}}{m_{2}} & \frac{-k_{3}}{m_{2}} & 0 \\
0 & 0 & 0 & 1 & 0 & -1 \\
0 & 0 & 0 & 0 & \frac{k_{3}}{m_{3}} & 0
\end{array}\right] \quad C=\left[\begin{array}{ccccccc}
1 & 0 & 0 & 0 & 0 & 0 \\
\frac{k_{1}}{m_{1}} & \frac{-c_{2}}{m_{1}} & \frac{-k_{2}}{m_{1}} & \frac{c_{2}}{m_{1}} & 0 & 0 \\
0 & 0 & 1 & 0 & 0 & 0 \\
0 & \frac{c_{2}}{m_{2}} & \frac{k_{2}}{m_{2}} & \frac{-c_{2}}{m_{2}} & \frac{-k_{3}}{m_{2}} & 0 \\
0 & 0 & 0 & 0 & 1 & 0 \\
0 & 0 & 0 & 0 & \frac{k_{3}}{m_{3}} & 0
\end{array}\right] \\
& B=D=\left[\begin{array}{lllllllllll}
0 & 0 & 0 & \frac{-1}{m_{2}} & 0 & \frac{1}{m_{3}}
\end{array}\right]^{T} \quad U=F_{D 3} \quad G=\left[\begin{array}{llllll}
1 & 0 & 0 & 0 & 0 & 0
\end{array}\right]^{T} \quad W=\dot{z}_{0}
\end{aligned}
$$

The aim of LQR control strategy in this study is to design a state-feedback regulator with output weighting to obtain the control force $U=-K X$ where $K$ is the optimized state-feedback gain matrix to minimize the quadratic cost function

$$
J=\int_{0}^{\infty}\left[q_{1} y_{1}^{2}+q_{2} y_{2}^{2}+q_{3} y_{3}^{2}+q_{4} y_{4}^{2}+q_{5} y_{5}^{2}+q_{6} y_{6}^{2}+U^{T} R U\right] d t=\int_{0}^{\infty}\left[Y^{T} Q Y+U^{T} R U\right] d t
$$

where $q_{1}, q_{2}, q_{3}, q_{4}, q_{5}, q_{6}$ are output weighting factors and $R$ is control weighting factor.

When the excitation vector $W=0$, Eq.(4) is

$\dot{X}=A X+B U$

In Ref [6], the study of LQR method proves that control effect in the condition of excitation $W=0$ have slightly difference from that in the condition of $W \neq 0$. In order to make the solving process of the control force simply, Eq.(7) are often used to determine the optimized control force which can be solved by using the Lqry function in Matlab control box.

Semi-Active Control Strategy. In Semi-Active Control system the control force is realized by a MRD and because the MRD can only supply a certain range of damping force which means that 
MRD can't be adjusted to the optimized the control force determine by LQR control strategy in any time. When the active optimized force is out of the range of MRD's damping force, MRD's damping force is adjusted to the criterion that make the damping most closely to active control force determined by LQR control strategy.

Experimental test shows that MRD's force-velocity behavior has an complicated nonlinear phenomenon, and several dynamic models of MR dampers have been presented. Among these models, Bingham model is simply and is used extensively. Bingham model is

$$
F_{D}=f_{c 3} \operatorname{sgn}\left(\dot{z}_{2}-\dot{z}_{3}\right)+c_{03}\left(\dot{z}_{2}-\dot{z}_{3}\right)
$$

where $F_{D}$ is MRD's damping force, $c_{03}$ is the damping coefficient and $f_{c 3}$ is Coulomb friction force which can be changed under different magnetic fields.

Supposing the maximum value of $f_{c 3}$ is $f_{c 3 \max }$ and the active control force determined by LQR control strategy is $F_{D 3}$, the criterion to determine MRD's force is as follows:

If $\dot{z}_{2}-\dot{z}_{3} \geq 0$ and if $f_{c 3 \max }+c_{03}\left(\dot{z}_{2}-\dot{z}_{3}\right) \geq F_{D 3} \geq c_{03}\left(\dot{z}_{2}-\dot{z}_{3}\right)$, then $F_{D}=F_{D 3}$

If $\dot{z}_{2}-\dot{z}_{3} \geq 0$ and if $F_{D 3}>f_{c 3 \max }+c_{0}\left(\dot{z}_{2}-\dot{z}_{3}\right)$, then $F_{D}=f_{c 3 \max }+c_{0}\left(\dot{z}_{2}-\dot{z}_{3}\right)$

If $\dot{z}_{2}-\dot{z}_{3} \geq 0$ and if $F_{D 3}<c_{03}\left(\dot{z}_{2}-\dot{z}_{3}\right)$ (including $\left.F_{D 3}<0\right)$, then $F_{D}=c_{03}\left(\dot{z}_{2}-\dot{z}_{3}\right)$

If $\dot{z}_{2}-\dot{z}_{3}<0$ and if $c_{03}\left(\dot{z}_{2}-\dot{z}_{3}\right) \geq F_{D 3} \geq-f_{c 3 \max }+c_{03}\left(\dot{z}_{2}-\dot{z}_{3}\right)$, then $F_{D}=F_{D 3}$

If $\dot{z}_{2}-\dot{z}_{3}<0$ and if $F_{D 3}<-f_{c 3 \max }+c_{03}\left(\dot{z}_{2}-\dot{z}_{3}\right)$, then $F_{D}=-f_{c 3 \max }+c_{03}\left(\dot{z}_{2}-\dot{z}_{3}\right)$

If $\dot{z}_{2}-\dot{z}_{3}<0$ and if $F_{D 3}>c_{03}\left(\dot{z}_{2}-\dot{z}_{3}\right)$ (including $F_{D 3}>0$ ), then $F_{D}=c_{03}\left(\dot{z}_{2}-\dot{z}_{3}\right)$

\section{Simulated results}

The simulation is carried out by MATLAB. For comparison, the effects of passive, active and semi-active control are all calculated. The parameters used in active and semi-active control simulation of the quarter car model are $m_{1}=40 \mathrm{~kg}, m_{2}=230 \mathrm{~kg}, m_{3}=70 \mathrm{~kg}, k_{1}=130 \mathrm{kN} / \mathrm{m}$, $k_{2}=20 \mathrm{kN} / \mathrm{m}, k_{3}=10 \mathrm{kN} / \mathrm{m}$ and $c_{2}=1300 \mathrm{~N} \cdot \mathrm{s} / \mathrm{m}$.In passive control system $c_{3}=300 \mathrm{~N} \cdot \mathrm{s} / \mathrm{m}$, the other parameters are the same as active and semi-active control.

Suppose the road excitation is sinusoidal and amplitude is $10 \mathrm{~mm}$, the standard deviation of the seat acceleration, vehicle body acceleration, tyre dynamic load under different exciting frequencies is showed as Figs.2-4.

Fig. 2 shows that in the frequency range lower than $30 \mathrm{rad} / \mathrm{s}$, compared to passive control, active and semi-active control can depress the seat acceleration effectively, the active control effect is better than semi-active control. But when the exciting frequency is larger 30rad $/ \mathrm{s}$, these controls has slightly difference.

Fig.3 shows that in the frequency range lower than the first natural frequency, three controls have slightly difference in vehicle body acceleration, between the first the second natural frequency, the acceleration under passive control is lower than active and semi-active control, and in the frequency upper than the second natural frequency, the acceleration under passive control is larger than active and semi-active control.

Fig. 4 shows that in the frequency range between $9.2 \mathrm{rad} / \mathrm{s}$ and $17.5 \mathrm{rad} / \mathrm{s}$, tyre dynamic load under passive control is lower than active and semi-active control, in lower frequency and in the frequency range upper than $17.5 \mathrm{rad} / \mathrm{s}$, these controls has slightly difference.

\section{Summary}

In order to improve the ride comfort, three seat suspension control system ie. passive, active and semi-active control has been studied. The LQR control strategy has been used to determine the active control force which is then matched by MRD in semi-active control by the criterion that make the MRD damping force most close to active control force.

A three degree of freedom quarter vehicle model has been used to calculate the control effect. From the simulation result it has been shown that semi-active control effect to depress seat acceleration is not as good as active control, but compared to passive control it can depress seat 
acceleration obviously. Besides the frequency range between the first and the second natural frequency, the vehicle body acceleration and the tyre dynamic load has slightly difference between the passive and semi-active control.

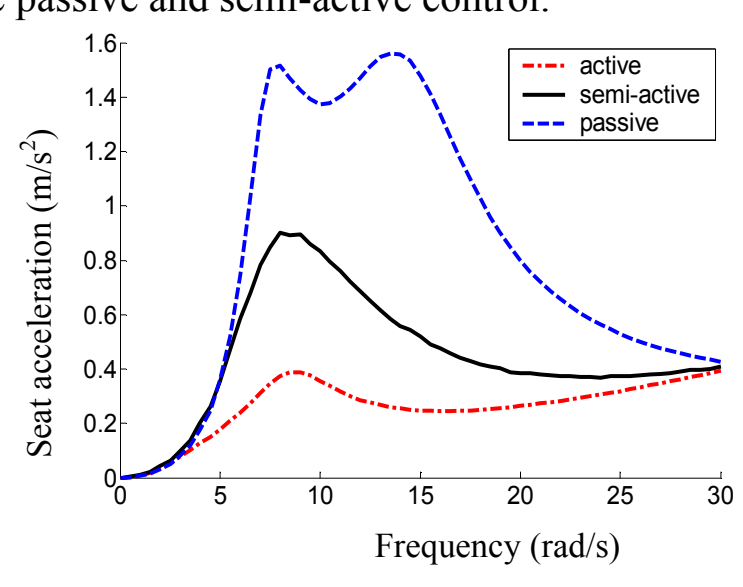

Fig. 2. Standard deviation of seat acceleration

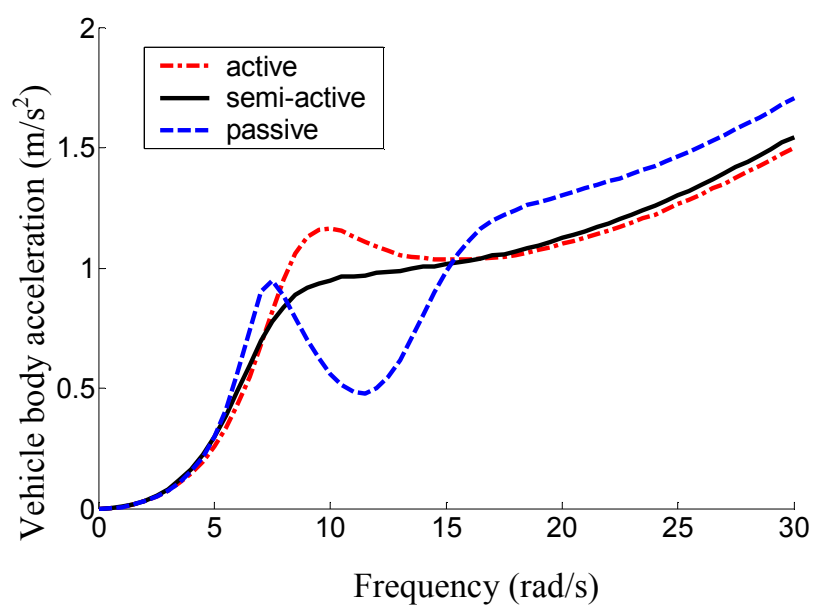

Fig. 3. Standard deviation of vehicle body acceleration

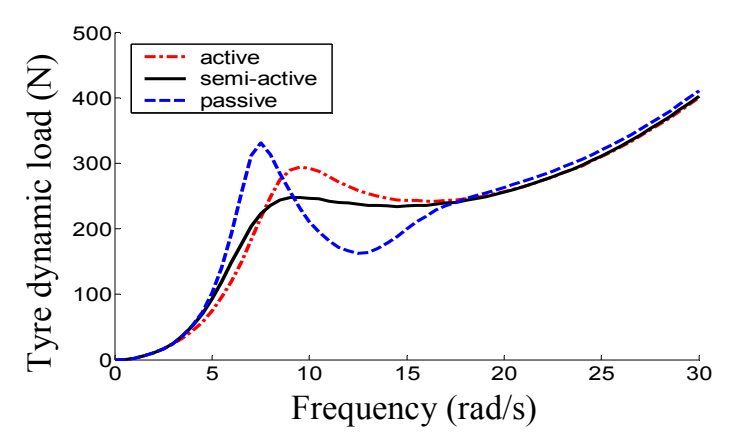

Fig. 4. Standard deviation of tyre dynamic load

\section{Acknowledgement}

This work is supported by the Program for Changjiang Scholars and Innovative Research Team in University (No. IRT0971) and Natural Science Funds for Distinguished Young Scholar of Hebei Province (No. E2010002047).

\section{References}

[1] R.S. Prabakar, C.Sujatha, S.Narayanan, Optimal semi-active preview control response of a half car vehicle model with magnetorheological damper, Journal of Sound and Vibration 326 (2009) 400-420.

[2] L.V.V. Gopala Rao, S. Narayanan, Sky-hook control of nonlinear quarter car model traversing rough road matching performance of LQR control, Journal of Sound and Vibration 323 (2009) $515-529$.

[3] G.Z.Yao, F.F.Yap, G.Chen, W.H.Li, S.H.Yeo, MR damper and its application for semi-active control of vehicle suspension system, Mechatronics 12 (2002) 963 - 973.

[4] C.-M. Lee, A.H. Bogatchenkov, V.N. Goverdovskiy, Y.V. Shynkarenko, A.I. Temnikov, Position control of seat suspension with minimum stiffness, Journal of Sound and Vibration 292 (2006) $435-442$.

[5] J. Swevers, C. Lauwerys, B.Vandersmissen, M. Maes, K.Reybrouck, P. Sas, A model-free control structure for the on-line tuning of the semi-active suspension of a passenger car, Mechanical Systems and Signal Processing 21 (2007) 1422 - 1436.

[6] Zhongquan Gu, Kougen Ma, Weidong Chen Write, Active Control of Vibration , first ed., National Defense Industry Press, Peking, 1997 